\title{
Investigation the Absorption Coefficient and Energy Band Gap of Wood-Polyester Composite Applying Optical Transmission Method
}

\author{
${ }^{1}$ Lazem Hassan Aboud, ${ }^{2}$ Shaymaa Al-Rubaye, ${ }^{2}$ Ehssan Al-Bermany and ${ }^{2}$ Kalid Haneen Abass \\ ${ }^{1}$ Department of Laser Physics, College of Science for Women, \\ ${ }^{2}$ Department of Physics, College of Education for Pure Sciences, \\ University of Babylon, Babylon, Iraq \\ lazemrasul@yahoo.com
}

\begin{abstract}
The optical properties of wood-polyester composites were studied using the measurement of changes in the UV-visible light absorption spectrum. This study aimed to motivate the feasibility of obtaining Wood-Polymer Composites (WPC) displaying a performance superior. Polymer composites exhibited a new UV-visible absorption band at a wavelength $(190 \mathrm{~nm})$ which is attributed to interchain interaction. The optical transmission method was successfully used to determine the absorption coefficient $(\bullet)$, the dielectric constant and the energy gap of four samples of wood-polyester composites. The results indicated a significant improvement of the investigated optical properties and showed a strongly dependent on the nature of the material and the radiation type.
\end{abstract}

Key words: Wood-polyester, polymer, UV-visible light, radiation, nature, energy gap

\section{INTRODUCTION}

The use of Wood Polymer Composite (WPC) has tremendously increased due to their different advantages (Ahmed et al., 2009). The improvement in adhesion among plastic materials as well as wood fibers is very important for obtaining better composites. Composite improves mechanical, thermal, optical and physiochemical properties remarkably compared to conventional composites (Aina and Owonibi, 2011; Al-Bermany, 2011).

These composites have been widely studied. The properties of WPC depend on the characteristics of matrix and fillers, the fractional composition of wood fillers, chemical interaction between wood fibres and polymer, humidity absorption and processing conditions. Many researchers have been worked to development new materials using biomass resources for reinforcement, based on the potential properties of polymers. Effective utilization of biomass resources has been considered an important recipe for environmental protection. Liquefaction of wood is a technique that converts lignocellulosic wastes such as sawdust and wood waste into substances soluble in commonly used organic solvent such as acetone, methyl alcohol or dioxane applying a liquefying agent such as phenols or polyhydric alcohol (Devi and Maji, 2007).

The action of the UV radiation is accompanied using the oxidation, therefore, the overall degradation reaction is one of photo-oxidation. The extent of degradation varies from location to location owing to differences in the intensity of radiation. This is considered very important in many applications because the degradation is reflected in the case of transparent compositions in a yellowing effect and generally, in a loss of mechanical properties such as a lower elongation at break and reduced impact strength (Doh et al., 2005; El-Raheem et al., 2012).

The study of UV rays effect on polymers has attracted considerable interest for many years. Spectroscopy is a fundamental part of such work. Polymers with pendant aromatic ring groups such as polyester are known to show a new fluorescence band at longer wavelength under irradiation due to the interaction between excited and ground state aromatic groups, i.e., the formation of intermolecular excimers. This phenomenon has been widely used as a powerful tool in polymer structure studies (Hadi et al., 2011)

The irradiation of polymers with ionizing radiations leads to a wide variety of changes in their physicochemical properties which can generally be traced back to the rearrangement taking place in the chemical structure of the polymer as a result of energy deposition (Helms et al., 2008).

\section{MATERIALS AND METHODS}

Experimental details: The wood polymer samples were prepared from $(5,10,15$ and $20 \mathrm{wt. \%})$. All these samples

Corresponding Author: Lazem Hassan Aboud, Department of Laser Physics, College of Science for Women, University of Babylon, Babylon, Iraq, lazemrasul@yahoo.com 
(a)

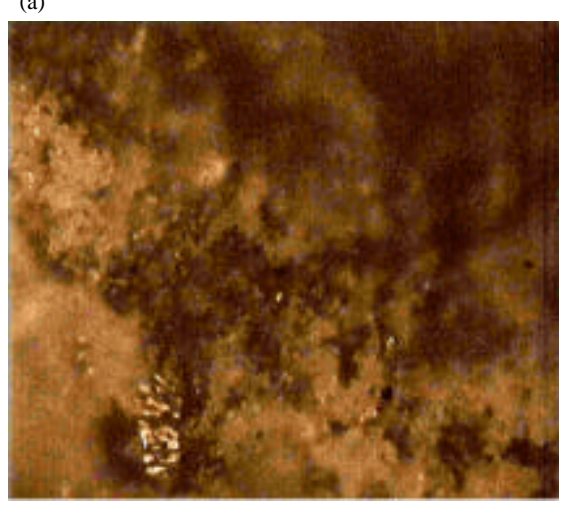

(b)

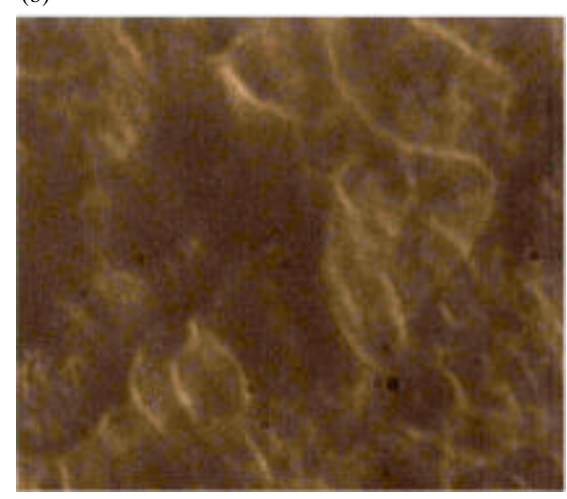

Fig. 1: Microscopic structure of surface for composite of polyester with $(\mathrm{NaCl})$ treatment observed by optic microscope $(150 \times)$ : a) Not confirmable by sawdust and b) Confirmable by sawdust

were subjected to UV-visible transmission and reflection studies using Shimadzu double-beam UV-vis spectrophotometer (UV-210 A) in the wavelength range $190-800 \mathrm{~nm}$, the method of measurement has been described elsewhere (Heskins and Guillet, 1970).

Preparation of wood sample: Woods were collected from the local forest. The sawdust was wetted with water where initially was washed with $1 \%$ soap solution, followed by a $1 \% \mathrm{NaOH}$ solution and later with cold distal water. The washed wood strips were dried using the oven at $110^{\circ} \mathrm{C}$ for $(24 \mathrm{~h})$ to prepare it for next stage. Then, sawdust was grinded in a mixer grinded to obtain the required degree of smoothness and sieved to obtain the particular size $(150 \mu \mathrm{m})$.

The mixing process took place by using an electrical mixer for $(5 \mathrm{~h})$. Then, the sawdust has been treated chemically in order to increase the surface roughness and the adhesion between sawdust and the polymer. The composite sheets were obtained by the compression moulding press at $150^{\circ} \mathrm{C}$ under pressure of $80 \mathrm{MPa}$.

The polymer used in this study was polyester types of unsaturated polyester resin have a density of $\left(1.1923 \mathrm{~g} / \mathrm{cm}^{3}\right)$ and low viscosity. This polymer was hardened by stimulated elements and accelerator, hard materials. Polymers are widely used in composites as fibres or as the matrix. More information about polymers can be found in the literature indicated (Kojima et al., 1993).

Optical calculation: Ultraviolet-visible absorbance was assumed to be zero above $700 \mathrm{~nm}$, therefore, the average sample absorbance between 700 and $800 \mathrm{~nm}$ was subtracted from the spectrum to correct for offsets due to instrument baseline drift, temperature, scattering and refractive effects. Absorbance units were converted to absorption coefficients follows (Kumbhakar et al., 2014):

$$
\alpha=2.303 \mathrm{~A} / 1
$$

Where:

- $=$ The absorption coefficient $\left(\mathrm{m}^{{ }^{1}}\right)$ for the solutions

$\mathrm{A}=$ The Absorbance for the solution

$1=$ The thickness of samples

The extinction coefficient is calculated by using the following Eq. 2 (Li et al., 1991):

$$
\mathrm{K}=\alpha \lambda / 4 \pi
$$

Ultraviolet-Visible (UV/Vis) spectroscopy has become an important tool to estimate the value of optical gap Energy $\left(\mathrm{E}_{\mathrm{g}}\right)$ in the polymer. The optical absorption edge can be correlated to optical gap Energy $\left(\mathrm{E}_{\mathrm{g}}\right)$ using Tauc's Eq. 3(Heskins and Guillet, 1970). The intersection of the extrapolated spectrum with the abscissa yields the optical gap Energy $\left(\mathrm{E}_{\mathrm{g}}\right)$. The Tauc's Eq. 3 is as follows (Li et al., 1991; Rizk et al., 2009) (Fig. 1):

$$
\alpha h v=A\left(h v-E_{g}\right)^{n}
$$

Where:

- $=$ The wavelength

$\mathrm{h}=\mathrm{A}$ Plank's constant $6.62617 \times 10^{-34} \mathrm{~J} \mathrm{~s}$

$\mathrm{c}=$ The speed of light $3 \times 10^{8} \mathrm{~m} / \mathrm{sec}$

\section{RESULTS AND DISCUSSION}

Figure 2 represents the UV-vis spectra of the wood-polyester composite. It is shown that the adding of the filler content to the polymer $(5,10,15$ and $20 \mathrm{wt} . \%)$ lead to increase the intensity of peak up to a maximum value at $10 \%$ wood. That suggests a uniform mixture of 


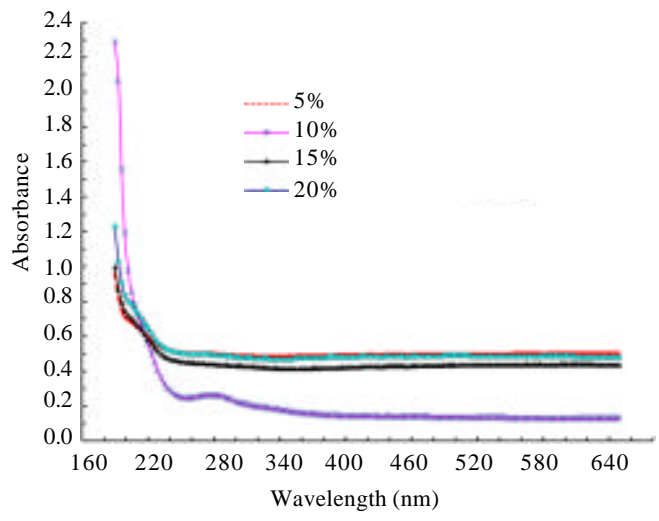

Fig. 2: UV/Vis spectra of wood-polyester composites

polymer with little variation in particle size. In the spectrum, the result may be attributed to the stretching of wood composites which indicated the formation of a bond between polymers and wood. These results indicate that the improvement in the interaction among the polymers caused by the addition of wood (Roppolo et al., 2016).

The UV-Vis spectra of wood-polyester composite showed the strong absorbance at $190 \mathrm{~nm}$. The absorption of light energy by polymeric materials in UV and visible regions involves the transition of electrons in $\mathrm{n}$ orbital from the ground state to higher energy state. The saturated compounds containing atoms with lone pairs (non-bonding electrons) are capable of $\mathrm{n} \bullet \mathrm{s}^{*}$ transitions. These transitions usually need less energy than $s \cdot s$ * transitions and can be initiated by light whose wavelength is in the range $150-250 \mathrm{~nm}$. The number of organic functional groups with $n \cdot \mathrm{s}^{*}$ peaks in the UV region is small (Heskins and Guillet, 1970).

The absorption coefficient - was determined for each sample using Eq. 1. It is clear that - must be a strong function of the energy h $\boldsymbol{h}^{-}$of the photons. Figure 3 shows that for $\mathrm{h} \bullet<\mathrm{E}_{\mathrm{g}}$, no electron-hole pairs can be created, the material is transparent and $\bullet$ is small. For $\mathrm{h} \bullet \mathrm{E}_{\mathrm{g}}$, the absorption should be strong (Rosa et al., 2010; Sliwa et al., 2012; Srivastava et al., 2010). Our results showed that the absorption coefficient - increased with increasing of the amount of wood; this was attributed to the stretching of composites (Sui et al., 2009).

In order to determine the optical band gap $\left(\mathrm{E}_{\mathrm{g}}\right)$ of the samples, the absorption data corresponding to the fundamental absorption edge as a function of wavelength was considered. These data were plotted in the form of $\left(\bullet h^{\bullet}\right)^{1 / 2}$ as a function of photon energy $\mathrm{h} \bullet$. The optical band gap values weredetermined from the intercepts of the plots of $(\bullet h \bullet)^{1 / 2}$ versus $(h \bullet)$ on $h \bullet$ axis as shown in

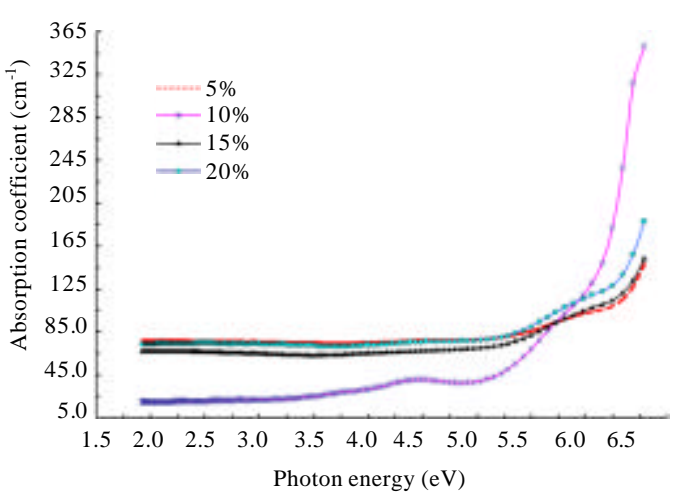

Fig. 3: The absorption coefficient for wood-polyester composition as a function of photon energy

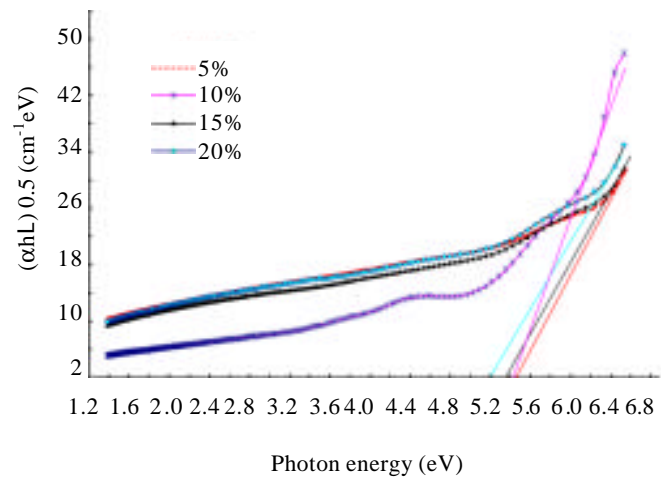

Fig. 4: Plot of $(\bullet h \bullet)$ 0.5. hu for wood-polyester composites

Fig. 3 (Helms et al., 2008; Rizk et al., 2009). Figure 4 reveals that the optical gap of the wood-polyester composites presented the values $5.47,5.44,5.38$ and $5.2 \mathrm{eV}$ for the sawdust concentrations $(5,10,15$ and $20 \%)$, respectively. Figure 4, all the composites showed a decreasing in the optical gap with each addition of wood concentrations of polyester. This behaviour may be attributed to a decrease in the packing density with increasing wood content of polymer (Rizk et al., 2009; Rosa et al., 2010; Tauc, 1972).

The variation of extinction coefficient $(\mathrm{k})$ with wavelength for wood-polyester composites shown in Fig. 5 indicating that the extinction coefficient increased with the increase of the weight percentage of the wood to the polyester and it is clear that the curves of the composites decreased with the increase of the incident wavelength until reach $250 \mathrm{eV}$ then the curves increases with increasing wavelength. These results can be described according to the random chain scission of several types of bonds. It occurs by a depolymerisation process which takes place through a chain scission mechanism (Zhang et al., 2009). 


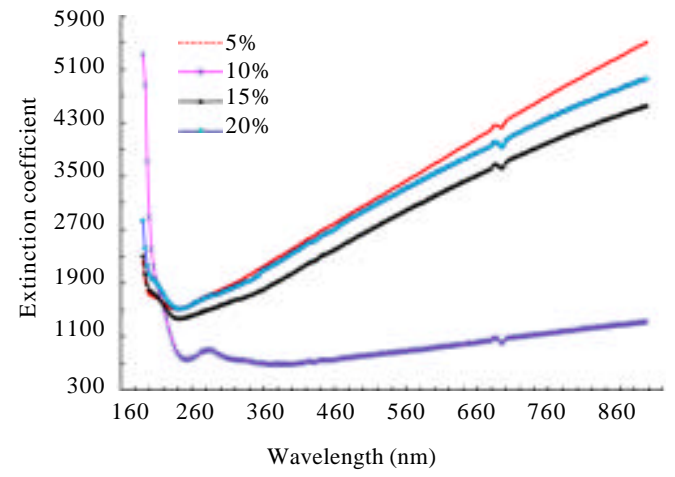

Fig. 5: The variation of extinctation coefficient for wood-polyester composites with incident wavelength

\section{CONCLUSION}

In this research, the optical method was applied to describe the measurement of wood-polyester composites which uses total reflectance of the polymer composites sample at a wavelength range 190-800 nm. The optical properties results of composites showed significantly changed using the modified wood. The absorption coefficient increased with increasing the ratio filler of content. The optical energy gap decreased with increasing the concentration of wood in polyester.

The extinction coefficient $(\mathrm{k})$ increased with the increaseof the weight percentage of the added wood to the polyester whereas it decreased with the increase of the incident wavelengthuntil reach $250 \mathrm{eV}$, then, increased after that. This result indicated that the doping atoms of wood modify the structure of the host polymer. The motivation for this study is to develop blends of these composites to produce a material with optimized optical properties and the effect of energy ion on the optical properties of the polymer blend that was studied in the present study.

\section{REFERENCES}

Ahmed, N.M., Z. Sauli, U. Hashim and Y. Al-Douri, 2009. Investigation of the absorption coefficient, refractive index, energy band gap and film thickness for $\mathrm{Al}_{0.11} \mathrm{Ga}_{0.89} \mathrm{~N}, \mathrm{Al}_{0.33} \mathrm{Ga}_{0.97}$ and $\mathrm{GaN}$ by optical transmission method. Int. J. Nanoelectron. Mater. , 2: 189-195.

Aina, D O. and K. Owonibi, 2011. Beekeeping prospects: Palynology and the environment. Adv. Applied Sci. Res, 2: 79-85.

Al-Bermany, E.D.J., 2011. Study some physical properties of post-irradiation for polystyrene dissolved in toluene. Am. J. Sci. Res., 29: 130-141.
Devi, R.R. and T.K. Maji, 2007. Effect of glycidyl methacrylate on the physical properties of wood-polymer composites. Polym. Compos., 28: $1-5$.

Doh, G.H., S.Y. Lee, I.A. Kang and Y.T. Kong, 2005. Thermal behavior of Liquefied Wood Polymer Composites (LWPC). Compos. Struct., 68: 103-108.

El-Raheem, M.M.A., M.S. Rasheedy, H.E. Ahmed, M.S.A. El-Aal and H.H. Al-Ofi et al., 2012. Characterization of thin films of Cd-doped $\mathrm{SnO}$ for optoelectronic applications. Adv. Appl. Sci. Res., 3: 227-234.

Hadi, S., A. Hashim and A. Jewad, 2011. Optical properties of (PVA-LiF) composites. Aust. J. Basic Applied Sci., 5: 2192-2195.

Helms, J.R., A. Stubbins, J.D. Ritchie, E.C. Minor and D.J. Kieber et al., 2008. Absorption spectral slopes and slope ratios as indicators of molecular weight, source and photobleaching of chromophoric dissolved organic matter. Limnol. Oceanogr., 53: 955-969.

Heskins, M. and J.E. Guillet, 1970. Photochemistry of ketone polymers III. Energy transfer in ethylene-carbon monoxide polymers. Macromol., 3: 224-231.

Kojima, Y., A. Usuki, M. Kawasumi, A. Okada and Y. Fukushima et al., 1993. Mechanical properties of nylon 6-clay hybrid. J. Mater. Res., 8: 11851189.

Kumbhakar, P., S.S. Ray and A.L. Stepanov, 2014. Optical properties of nanoparticles and nanocomposites. J. Nanomater., 2014: 1-2.

Li, T., C. Zhou and M. Jiang, 1991. UV absorption spectra of polystyrene. Polym. Bull., 25: 211-216.

Rizk, R.A.M., A.M. Abdul-Kader, Z.I. Ali and M. Ali, 2009. Effect of ion bombardment on the optical properties of LDPE/EPDM polymer blends. Vac., 83: 805-808.

Roppolo, I., M. Sangermano and A. Chiolerio, 2016. Optical Properties of Polymer Nanocomposites. In: Functional and Physical Properties of Polymer Nanocomposites, Dasari, A. and J. Njuguna (Eds.). John Wiley \& Sons Inc., Hoboken, New Jersey, USA., ISBN:9781118542323, pp: 139-157.

Rosa, I.M.D., J.M. Kenny, D. Puglia, C. Santulli and F. Sarasini, 2010. Morphological, thermal and mechanical characterization of okra (Abelmoschus esculentus) fibres as potential reinforcement in polymer composites. Compos. Sci. Technol., 70: 116-122. 
Sliwa, F., G. Marin, F., Charrier and F. Malet, 2012. A new generation of wood polymer composite with improved thermal stability. Polym. Degrad. Stab., 97: 496-503.

Srivastava, A., V. Singh, P. Aggarwal, F. Schneeweiss and U.W. Scherer et al., 2010. Optical studies of insulating polymers for radiation dose monitoring. Indian J. Pure Appl. Phys., 48: 782-786.
Sui, G., M.A. Fuqua, C.A. Ulven and W.H. Zhong, 2009. A plant fiber reinforced polymer composite prepared by a twin-screw extruder. Bioresour. Technol., 100 : 1246-1251.

Tauc, J., 1972. States in the gap. J. Non-Crystalline Solids, 8-10: 569-585.

Zhang, K., B.J. Park, F.F. Fang and H.J. Choi, 2009. Sonochemical preparation of polymer nanocomposites. Mol., 14: 2095-2110. 